\title{
MEASUREMENT OF THE DIELECTRIC PROPERTIES OF LIQUID CRYSTAL MATERIAL FOR MICROWAVE APPLICATIONS
}

Juan R. Sánchez ${ }^{1}$, Vicente Nova ${ }^{2}$, Carmen Bachiller ${ }^{2}$, Belén Villacampa ${ }^{3}$, Alberto de la $\mathrm{Rua}^{4}$, Rainer Kronberger ${ }^{4}$, Felipe Peñaranda ${ }^{1}$, and Vicente E. Boria ${ }^{2}$

${ }^{1}$ ITACA, Universitat Politècnica de València, Camino de Vera s/n, Valencia, Spain.

${ }^{2}$ ITEAM, Universitat Politècnica de València, Camino de Vera $s / n$, Valencia, Spain.

${ }^{3}$ Dto de Fisica de la Materia Condensada. Universidad de Zaragoza. Zaragoza, Spain.

${ }^{4}$ High Frequency Laboratory. TH Köln,. Betzdorfer Str. 2, Koeln, Germany.

Email:jrsanchez@itaca.upv.es

Keywords: dielectric characterization, liquid crystal, microwave, split-cylinder resonator.

\section{Introduction}

The use of Liquid Crystal (LC) in telecommunications for developing reconfigurable microwave devices is spreading [1-4]. LCs anisotropic materials with properties between crystalline solids and liquids. Their rod-like molecules present nematic phase, having orientational order but no positional one. The long axis of the molecules has a preferred position, which can be changed by applying an external electric or magnetic field.

In the macroscopic scale, the direction of the molecules is represented by a vector called director $\vec{n}$, which is parallel to the longitudinal axis of the LC molecules. The dielectric permittivity values of the nematic LCs are defined by the angle formed between the director vector and an external RF electric field $\vec{E}$. The values represented by $\varepsilon_{r \perp}$ and $\varepsilon_{r \|}$ are, respectively, the permittivity values when $\vec{n}$ is perpendicular and parallel to $\vec{E}$ [5]. Moreover, loss tangents $\tan \delta_{\perp}$ and $\tan \delta_{||}$are also defined corresponding to those directions.

Different methods can be used for this characterization. On the one hand, transmission line methods developed at millimiter-wave and microwave frequencies [6-8]. On the other hand, resonant methods, which can obtain dielectric properties at single frequency points have been also developed [9-12]. However, all of them need to apply an electric or magnetic field for measuring both polarization of the LC molecules. In this paper, it is proposed an alternative for measuring parallel and perpendicular dielectric properties without any external electromagnetic field. It is based on the split-cylinder resonator method.

\section{Method description}

The split-cylinder resonator consists of two cylindrical halves, where the sample is placed between them. The empty resonator has a specific resonance frequency and quality factor (Q-factor), and when the sample is placed in the middle, these parameters change. The relative change between the resonator without and with sample allows to calculate the dielectric permittivity and the loss tangent of the material. In the case of a LC material, the samples are confined in a cell formed by two glasses and the LC is sited between them as shown in Fig.1. To form the cell, firstly an alignment surface must be created in the inner side of the each glass. This surface consists of microgrooves created by depositing a polyimide layer and afterwards rubbing with a natural velvet. This will provoke the LC molecules to be aligned in the microgrooves direction. Next, the glasses are adhered by using four separators in the corners and the LC is placed in the gap between them filling the cell by capillarity. 
This process allows to obtain a cell formed by three layers (two glasses and the LC sample material). For this reason, classical methods for characterization [13-14] have to be modified for extracting the dielectric properties of the LC material sample placed between the two glasses. The glasses are characterized following the classical methods, therefore for each cell, the properties of the glasses are known. Thus, the modal analysis method described [1517] is used, where the structure is divided in canonical circuital elements and the only unknown is the dielectric properties of the LC material.

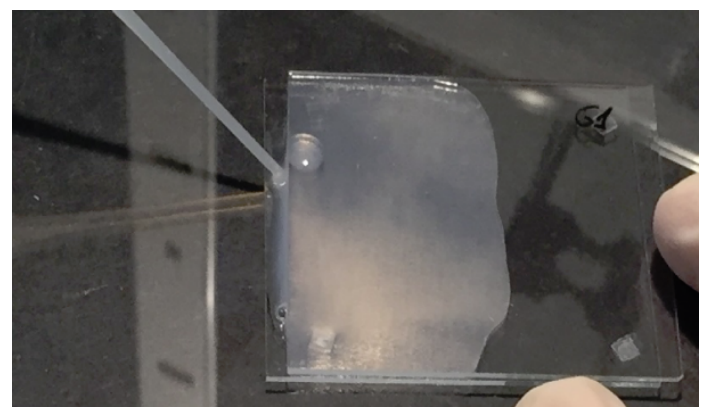

Fig. 1. Filling of a Liquid Crystal cell by capillarity.

The chosen working mode of the resonator is based on the field distribution. Mode $\mathrm{TE}_{111}$ has a quasi-straight field lines distribution, allowing to measure the two polarizations of the molecules of LC just rotating $90^{\circ}$ the cell inside the cavity without applying any external field, as shown in Fig. 2.

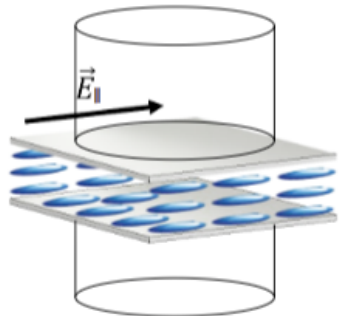

(a)

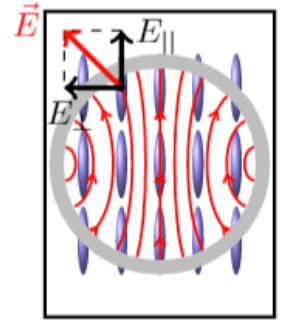

(c)

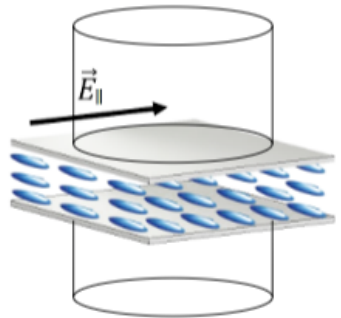

(b)

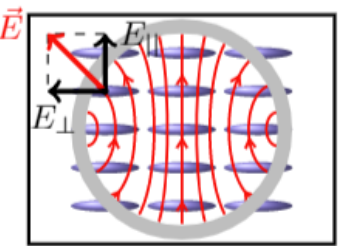

(d)

Fig. 2. (a) Placement of LC cell is Position 1. (b) Placement of LC cell is Position 2.

(c) E-field and LC molecules in Position 1. (d) E-field and LC molecules in Position 2. 
However, as shown in Fig. 1, the non-ideal straight field distribution, position 1 and 2 of the LC cell do not correspond to the parallel and perpendicular permittivity directly. The values can be extracted from the following equations:

$$
\begin{aligned}
& \varepsilon_{\text {rpos } 1} \iint|\vec{E}| d S=\varepsilon_{r \perp} \iint E_{\perp} d S+\varepsilon_{r||} \iint E_{||} d S \\
& \varepsilon_{\text {rpos } 2} \iint|\vec{E}| d S=\varepsilon_{r \|} \iint E_{\perp} d S+\varepsilon_{r \perp \mid} \iint E_{||} d S
\end{aligned}
$$

where:

- $\quad \varepsilon_{\text {rpos } 1}$ and $\varepsilon_{\text {rpos } 2}$ are the extracted complex permittivities with the cells in position 1 and 2.

- $\quad|\vec{E}|$ is the module of the electric field calculated in each point of the cell.

- $\quad E_{\|}$is the component of the electric field in parallel direction.

- $\quad E_{\perp}$ is the component of the electric field in the perpendicular direction.

\section{Results}

For performing the measurement a set-up composed of a VNA and a split-cylinder resonator is used, as shown in Figure 9. Four different nematic LCs have been prepared and measured by using different cells: QYPD036, QYPD142, and QYPD193 from Qingdao QY Liquid Crystal Co and GT3-23002 from Merck. The measurements have been carried out at two different frequencies $(5 \mathrm{GHz}$ and $11 \mathrm{GHz})$ at room temperature $\left(25^{\circ} \mathrm{C}\right)$.

The resonance frequency and the Q-factor for positions 1 and 2 have been measured for each LC cell. These measurements have been performed several times in order to extract an error value. With these values and following the method explained above, the complex dielectric permittivity values are obtained. Then, the transformation explained in (1) is applied to obtain the parallel and perpendicular dielectric permittivity and the loss tangent.

There are some error sources that affect the extraction of the complex permittivity: the features of the cylinder (radius, height, conductivity, and feeding loops), the measured EM response (resonance frequency and quality factor), the thickness of the glasses and the thickness of liquid crystal. The impact of the cylinder dimensions is calibrated and compensated with a first measurement of the empty cylinder. However, the tolerance in the measurement of the other parameters is translated into a set of uncertainties $\left(\sigma_{i}\right)$ and their error contribution to the complex permittivity results is quantified. Then, if the error contribution of these parameters is independent and known, the total uncertainty of the complex dielectric permittivity can be expressed as the quadrature sum [18] described in equation (2). Finally, the complex permittivity error can be easily propagated to obtain the loss tangent uncertainty.

$$
\sigma_{i}=\left(\sum\left|\frac{\delta \varepsilon}{\delta \text { error }}\right| \sigma_{\text {error }}^{2}\right)^{1 / 2}
$$

The results corresponding to $5 \mathrm{GHz}$ and $11 \mathrm{GHz}$ are presented in Tables 1 and 2, respectively. The highest value of anisotropy for the Qingdao LCs family, is 0.81 at $5 \mathrm{GHz}$ (QYPD193) and 0.98 at $11 \mathrm{GHz}$ (QYPD036). The highest values of the loss tangents are 
also recorded for these two materials. Therefore, choosing an LC for designing a reconfigurable microwave device must be a trade-off between the dielectric anisotropy and the related loss, depending on the required specifications.

Table 1. Dielectric properties of LCs at $5 \mathrm{GHz}$.

\begin{tabular}{ccccc}
\hline LC & $\varepsilon_{r \|}$ & $\tan _{\|} \mathrm{x} 1000$ & $\varepsilon_{r \perp}$ & $\tan _{\perp} \mathrm{x} 1000$ \\
\hline QYPD036 & $3.08 \pm 0.19$ & $17.06 \pm 2.08$ & $2.28 \pm 0.15$ & $75.45 \pm 7.89$ \\
QYPD142 & $2.72 \pm 0.17$ & $7.95 \pm 1.09$ & $2.27 \pm 0.16$ & $58.51 \pm 7.20$ \\
QYPD193 & $3.03 \pm 0.19$ & $7.15 \pm 0.97$ & $2.22 \pm 0.15$ & $91.90 \pm 7.55$ \\
GT3-23002 & $2.59 \pm 0.15$ & $5.13 \pm 1.79$ & $1.94 \pm 0.14$ & $39.33 \pm 10.90$ \\
\hline
\end{tabular}

Table 2. Dielectric properties of LCs at $11 \mathrm{GHz}$.

\begin{tabular}{ccccc}
\hline LC & $\varepsilon_{r \|}$ & $\tan _{\|} \times 1000$ & $\varepsilon_{r \perp}$ & $\tan \delta_{\perp} \times 1000$ \\
\hline QYPD036 & $3.22 \pm 0.19$ & $3.95 \pm 0.67$ & $2.24 \pm 0.15$ & $57.51 \pm 6.80$ \\
QYPD142 & $3.05 \pm 0.18$ & $5.80 \pm 1.06$ & $2.36 \pm 0.16$ & $40.88 \pm 4.92$ \\
QYPD193 & $3.26 \pm 0.20$ & $1.97 \pm 0.11$ & $2.40 \pm 0.17$ & $63.31 \pm 4.40$ \\
GT3-23002 & $3.25 \pm 0.21$ & $2.50 \pm 0.83$ & $2.35 \pm 0.17$ & $22.05 \pm 3.81$ \\
\hline
\end{tabular}

\section{Conclusions}

In this work, the split-cylinder resonator method has been used for the characterization of LCs at microwave frequencies. The method is based on the measurement of the resonance frequency and quality factor of the two states of the LC molecules. To achieve these two states, no electric or magnetic fields are needed, the cell must be turned $90^{\circ}$ inside the cavity. The dielectric properties (permittivity and loss tangent) of four different LC samples, GT323002 from Merck and QYPD193, QYPD142, and QYPD036 from Qingdao QY Liquid Crystal Co, have been obtained. The highest values of the dielectric anisotropy are presented for the samples QYPD036 and QYPD193, together with the highest values of the corresponding loss tangent parameters. Furthermore, it is observed for all the LCs that the loss tangent decreases and the dielectric anisotropy increases at higher frequencies. This must be taken into account in the development of reconfigurable microwave devices.

\section{References}

[1] M. Yazdanpanahi and D. Mirshekar-Syahkal, "Millimeter-wave liquid-crystal-based tunable bandpass filter," in 2012 IEEE Radio and Wireless Symp., Jan 2012, pp. 139142 .

[2] M. Jost, C. Weickhmann, T. Franke, A. E. Prasetiadi, W. Hu, M. Nickel,O. H. Karabey, and R. Jakoby, "Tunable hollow waveguide devices for space applications based on liquid crystal," in 2015 SBMO/IEEE MTT-S Int. Microw. and Optoelectronics Conf. (IMOC), Nov 2015, pp. 1-5.

[3] S. Strunck, O. H. Karabey, C. Weickhmann, A. Gaebler, and R. Jakoby, “Continuously tunable phase shifters for phased arrays based on liquid crystal technology," in 2013 IEEE Int. Symp. on Phased Array Systems and Technol., Oct 2013, pp. 82-88.

[4] K. Wang and $\mathrm{K}$. Wu, "Liquid crystal enabled substrate integrated waveguide variable phase shifter for millimeter-wave application at $60 \mathrm{GHz}$ and beyond," in2015 IEEE MTT-S Int. Microw. Symp., May 2015,pp. 1-4. 
[5] O. H. Karabey, Electronic Beam Steering and Polarization Agile Planar Antennas in Liquid Crystal Technology (Springer Theses).Springer, 2013.

[6] S. Mueller, A. Penirschke, C. Damm, P. Scheele, M. Wittek, C. Weil, and R. Jakoby, "Broad-band microwave characterization of liquid crystals using a temperaturecontrolled coaxial transmission line,'IEEE Trans.Microw. Theory Techn., vol. 53, no. 6, pp. 1937-1945, June 2005.

[7] S. Mueller, M. Koeberle, F. Goelden, A. Penirschke, A. Gaebler, A. La-panik, W. Haase, and R. Jakoby, "W-band characterization of anisotropic liquid crystals at room temperature," in2008 38th Eur. Microw. Conf., Oct 2008, pp. 119-122.

[8] S. Bulja, D. Mirshekar-Syahkal, R. James, S. E. Day, and F. A.Fernandez, "Measurement of dielectric properties of nematic liquid crystals at millimeter wavelength, "IEEE Trans. Microw. Theory and Techn., vol. 58, no. 12, pp. 3493-3501, Dec 2010.

[9] C. V. L.F. Chen, C.K. Ong and V. Varadan, "Chapter 5 - Resonator Methods," in Microwave Electronics: Measurement and Materials Characterization. Hoboken, UK: John Wiley \& Sons, Incorporated, 2004.

[10] A. Penirschke, S. Muller, P. Scheele, C. Weil, M. Wittek, C. Hock, and R. Jakoby, "Cavity perturbation method for characterization of liquid crystals up to $35 \mathrm{GHz}$," in34th Eur. Microw. Conf., 2004, vol. 2, Oct2004, pp. 545-548.

[11] R. Kowerdziej, J. Krupka, E. Nowinowski-Kruszelnicki, M. Olifierczuk, and J. Parka, "Microwave complex permittivity of voltage-tunable nematic liquid crystals measured in high resistivity silicon transducers," Applied Physics Letters, vol. 102, no. 10, p. 102904, 2013. [Online].Available: https://doi.org/10.1063/1.4795534

[12] R. Kowerdziej, J. Parka, J. Krupka, M. Olifierczuk, E. Nowinowski-Kruszelnicki, L. Jaroszewicz, and O. Chojnowska, "Dielectric properties of highly anisotropic nematic liquid crystals for tunable microwave components," Applied Physics Letters, vol. 103, no. 17, p. 172902, 2013. [Online]. Available: https://doi.org/10.1063/1.4826504

[13] G. Kent, "Nondestructive permittivity measurement of substrates," IEEE Trans. Instrum. Meas., vol. 45, no. 1, pp. 102-106, Feb 1996.

[14] M. D. Janezic and J. Baker-Jarvis, "Full-wave analysis of a split-cylinder resonator for nondestructive permittivity measurements,'IEEE Trans.Microw. Theory Techn., vol. 47, no. 10, pp. 2014-2020, Oct 1999.

[15] F. L. Penaranda-Foix and J. M. Catala-Civera, "Circuital analysis of cylindrical structures applied to the electromagnetic resolution of resonant cavities," in Passive Microwave Components and Antennas,V. Zhurbenko, Ed.Rijeka: IntechOpen, 2010, ch. 7. [Online].Available: https://doi.org/10.5772/9400

[16] F. L. Penaranda-Foix, M. D. Janezic, J. M. Catala-Civera, and A. J. Canos, "Fullwave analysis of dielectric-loaded cylindrical waveguides and cavities using a new four-port ring network, 'IEEE Trans. Microw. Theory Techn., vol. 60, no. 9, pp. 2730 2740, Sept 2012.

[17] D. Marques-Villarroya, F. L. Penaranda-Foix, B. Garcia-Banos, J. M.Catala-Civera, and J. D. Gutierrez-Cano, "Enhanced full-wave circuit analysis for modeling of a split cylinder resonator," IEEE Trans. Microw. Theory Techn., vol. 65, no. 4, pp. 11911202, April 2017.

[18] J. Taylor, An introduction to Error Analysis. Sausalto, CA, USA: University Science Books, 1997. 